\title{
Free induction signal from biexcitons and bound excitons
}

\author{
Emmanuel I. Rashba \\ Department of Physics, University of Utah, Salt Lake City, Utah 84112 \\ and L. D. Landau Institute for Theoretical Physics, Moscow 117940, Russia
}

(23 December 1996)

\begin{abstract}
A theory of the free induction signal from biexcitons and bound excitons is presented. The simultaneous existence of the exciton continuum and a bound state is shown to result in a new type of time dependence of the free induction. The optically detected signal increases in time and oscillates with increasing amplitude until damped by radiative or dephasing processes. Radiative decay is anomalously fast and can result in strong picosecond pulses. The expanding area of a coherent exciton polarization (inflating antenna), produced by the exciting pulse, is the underlying physical mechanism. The developed formalism can be applied to different biexciton transients.
\end{abstract}

71.35.Cc, 42.50.Md, 78.47.+p

Typeset using REVTEX 


\section{INTRODUCTION}

Ultrafast spectroscopy of excitons in the time domain 1 has proven to be a powerful tool to probe quantum coherence of exciton states, which was originally studied by polarization of the stationary emission. 2 Most of the experimental data were taken from GaAs quantum wells, however, some experiments were performed with bulk excitons. Quantum beats in different response functions provide a manifestation of the coherence driven by external fields. These beats appear when several states having close energies are excited simultaneously. Quantum beats were observed with magnetically split exciton levels, different confinement energies, and with biexcitons and a two-exciton continuum. 7.8 Two last systems have much in common since they possess both a continuous spectrum and a discrete level. They will be of principal importance for us in what follows.

Experimental data provide convincing evidence of a strong effect of the exciton-exciton and exciton-free-carrier interaction on nonlinear response functions 9.10 However, there could not be universal approach to the theoretical treatment of this nonlinear many-electron problem. The physical patterns are rather different in different ranges of the parameter values, therefore, the theoretical approaches also should be different. When the intensity of a pump is high and the nonradiative dephasing time $\tau$ is short, the Hartree-Fock approximation works rather well. This approach was advanced by Schmitt-Rink and Chemla11 and has been developed in a number of papers. It is based on the semiconductor Bloch equations 12,13 and allows a generalization accounting for two-exciton correlations. 14 Time evolution of wave packets produced by the pump also can be followed.5 It is a distinctive feature of this approach that the basis of the exciton and electron-hole pair states is restricted by excitations strongly coupled to the pump, i.e., possessing the momenta of the exciting electromagnetic waves. This restriction is the basic limitation of the powerful Hartree-Fock method. A different approach is used in the opposite limit of low exciton densities, when the interaction of biexcitons can be neglected and the dephasing time $\tau$ is long. In this limit biexcitons can be 
considered as noninteracting particles, and the problem can be reduced to the dynamics of an exciton molecule in external fields. Even a simple four-level biexciton energy scheme16 17 turned out to be very successful. In the framework of this scheme the biexciton continuum is substituted by a single two-exciton state. The four-level scheme allowed one to explain the existence of beats, having a period of inverse biexciton binding energy, in different nonlinear phenomena. More recently, Wang et al. 8 have discussed the effect of the biexciton continuum on initial biexciton transients. These authors neglected the exciton-exciton interaction in the biexciton continuum and investigated quantum evolution of the relative momentum of two excitons in a molecule. Our paper is related to the approach advanced in Refs. 16.17, and 8 . Developing a theory that allows for a consistent account of the biexciton continuum and the exciton-exciton interaction is the main objective of the paper. We show that the interaction of excitons and their dynamics are of critical importance for biexciton transients. Our results are primary developed for an exciton in the field of a defect. This problem is of intrinsic importance and is actively studied. Fast early-time evolution of the Rayleigh signal from inhomogeneously broadened exciton levels was recently investigated both theoretically and experimentally. 18

The traditional approach to quantum beats is based on the energy spectrum comprising few, usually two, discrete energy levels. This approach can be applied to beats between heavy- and light-hole excitons because of the momentum conservation and absence of the interaction between these excitons. However, the biexciton and bound exciton problems are more involved. Indeed, energy spectra of these systems include the two-exciton and single-exciton continuum, respectively. For example, for a two-exciton system it is the exciton-exciton interaction that supports the two-photon coherence, and the lower part of the continuum with the width of about several biexciton binding energies, $\varepsilon_{b}$, contributes to the coherent polarization along with the bound biexciton state. In addition to the theoretical arguments, the experimental data provide weighty, although indirect, evidence of the role of the two-exciton continuum. Indeed, it was shown 16 that the four-level biexciton energy scheme can be brought into agreement with experimental data only if the enhance- 
ment factor typical of giant oscillator strengths 19 is invoked. Therefore, frequencies of allowed transitions are distributed continuously and the spread of the frequencies is about $\varepsilon_{b}$. Naive consideration suggests that such an energy spectrum should result in beats having a frequency of about $\varepsilon_{b}$ and showing fast nondissipative decay because of accumulating phase differences between different modes. It turns out that the actual physical picture is quite different.

In this paper we present an exact solution for a free induction signal excited by a singleside exponential pulse, $t<0$, in a nondissipative system with a biexciton nonlinearity. This signal can be observed, e.g., as a resonance fluorescence at positive times, $t>0$. The special shape of the pulse simplifies calculations but does not influence the basic results. The contribution of the two-exciton continuum is consistently taken into account.22 With such an approach, free induction, i.e., free oscillations of a two-exciton wave function $\Psi(t)$ for $t>0$, includes two modes. There exists a beating mode describing undamped beats whose frequency is equal to $\varepsilon_{b}$. Damping of this mode is controlled by the mechanisms other than the exciton dispersion (radiative decay, dephasing, polariton effects, etc.). There exists also a growing mode whose amplitude increases linearly with time $t$ and whose carrier frequency equals the energy of the bottom of the two-exciton continuum. The growing mode is inherent in interacting systems possessing a continuous spectrum. It is the inflation in a real space of the wave packet created by the pulse that manifests itself in this mode. The packet is termed an inflating antenna in what follows. The two modes result in an optically detected free induction signal that (i) increases with $t$, (ii) has monotonic and oscillating parts, and (iii) results in ultrafast radiative decay. The same modes exist for excitons bound to impurities. Both modes originate from the analytical structure of the twoexciton Green function, which is specific for Hamiltonians with a continuous spectrum and violated momentum conservation. We expect that these modes contribute also to different nonlinear processes, including multiple-impulse processes, and that the developed technique is of general applicability. 


\section{BOUND EXCITONS: GENERAL FORMALISM}

To make clear the basic idea and account rigorously for analytical properties of the exciton Green functions, we develop an exactly soluble model. To this end we neglect polariton effects, 23 which will be briefly discussed in Sec. \, and dephasing. We also neglect the dependence of the scattering amplitude on the light polarization because it is sensitive

to the band structure, geometry, etc. 10 , Excitons are considered stable particles without internal degrees of freedom. It is convenient to start with the bound exciton problem. The free induction signal from bound excitons can be found in the framework of the linear response approach. Therefore, the specific spectral properties of the inflating antenna can be completely disentangled from nonlinear phenomena.

If the electromagnetic wave,

$$
\mathbf{E}_{\mathbf{q}}(\mathbf{r}, t) \propto \exp \{i(\mathbf{q} \cdot \mathbf{r}-\omega t)+\alpha t\}, \quad \alpha>0
$$

is incident upon a crystal at $-\infty<t<0$, the exciton wave function $\Psi_{\mathbf{q}}\left(\mathbf{q}^{\prime}, t=0\right)$ can be calculated as a linear response to this perturbation. Free evolution of $\Psi_{\mathbf{q}}\left(\mathbf{q}^{\prime}, t=0\right)$ determines the wave function $\Psi_{\mathbf{q}}\left(\mathbf{q}^{\prime}, t\right)$ at any instant $t, t>0$ :

$$
\Psi_{\mathbf{q}}\left(\mathbf{q}^{\prime}, t\right)=(i M / \sqrt{v}) A_{\mathbf{q}^{\prime} \mathbf{q}}(t), \quad A_{\mathbf{q}^{\prime} \mathbf{q}}(t)=\sum_{j} \frac{\psi_{j}\left(\mathbf{q}^{\prime}\right) \bar{\psi}_{j}(\mathbf{q})}{\omega-E_{j}+i \alpha} \mathrm{e}^{-i E_{j} t}
$$

Here $\Psi_{\mathbf{q}}\left(\mathbf{q}^{\prime}, t\right)$ and $\psi_{j}\left(\mathbf{q}^{\prime}\right)$ are, respectively, time-dependent and stationary exciton wave functions in the momentum representation. The subscript $j \geq 1$ numerates single-exciton states, bound and free ones. The ground-state energy of the crystal is chosen as the origin, $E_{0}=0 ; \hbar=1$. The coefficient $M$ is the matrix element, per unit cell, of the perturbation produced by the field $\mathbf{E}_{\mathbf{q}}(\mathbf{r}, t)$, and $v$ is the unit cell volume. The momenta $\mathbf{q}$ and $\mathbf{q}^{\prime}$, which are of importance for optical experiments, are small and will be neglected in the final results. Equation (2) does not take into account the radiative decay of the wave packet. The role of this process will be discussed in Sec. [II] below.

The amplitude $A_{\mathbf{q}^{\prime} \mathbf{q}}(t)$ describes free precession of $\Psi_{\mathbf{q}}\left(\mathbf{q}^{\prime}, t\right)$ for positive times. For $t=0$, $A_{\mathbf{q}^{\prime} \mathbf{q}}(t=0)$ coincides with the retarded exciton Green function 


$$
G_{\mathbf{q}^{\prime} \mathbf{q}}(\omega)=\sum_{j} \psi_{j}\left(\mathbf{q}^{\prime}\right) \bar{\psi}_{j}(\mathbf{q}) /\left(\omega-E_{j}+i 0\right)
$$

if the argument $\omega$ is substituted by $\omega+i \alpha$. For arbitraty $t$, functions $A_{\mathbf{q}^{\prime} \mathbf{q}}(t)$ and $G_{\mathbf{q}^{\prime} \mathbf{q}}(\omega)$ are related by the equation

$$
A_{\mathbf{q}^{\prime} \mathbf{q}}(t)=\frac{1}{2 \pi i} \int_{-\infty}^{\infty} d \omega^{\prime} \frac{\exp \left(-i \omega^{\prime} t\right)}{\omega^{\prime}-\omega-i \alpha} G_{\mathbf{q}^{\prime} \mathbf{q}}\left(\omega^{\prime}\right)
$$

Equation (4) can be checked by employing Eq. (3), closing the integration path in the lower complex half-plane, and calculating residues in the poles of $G_{\mathbf{q}^{\prime} \mathbf{q}}\left(\omega^{\prime}\right)$.

Subsequent transformations of $A_{\mathbf{q}^{\prime} \mathbf{q}}(t)$ are based on the introduction of the scattering operator $25 \hat{\mathcal{T}}(\omega)$

$$
G_{\mathbf{q}^{\prime} \mathbf{q}}(\omega)=G_{\mathbf{q}}^{0}(\omega) \delta_{\mathbf{q}^{\prime} \mathbf{q}}+G_{\mathbf{q}^{\prime}}^{0}(\omega) \mathcal{T}_{\mathbf{q}^{\prime} \mathbf{q}}(\omega) G_{\mathbf{q}}^{0}(\omega)
$$

where $G_{\mathbf{q}}^{0}(\omega)=[\omega-\varepsilon(\mathbf{q})+i 0]^{-1}$ is a free-exciton Green function. Only the second term of Eq. (5) contributes to $A_{\mathbf{q}^{\prime} \mathbf{q}}(t)$ for $\mathbf{q}^{\prime} \neq \mathbf{q}$ and will be retained below. It is an important property of this term that it includes a product of two $G^{0}$ functions. Their poles nearly coincide for $\mathbf{q} \approx \mathbf{q}^{\prime}$. This property strongly influences the subsequent results. It is convenient to split the scattering operator $\hat{\mathcal{T}}$ into two terms as

$$
\hat{\mathcal{T}}(\omega)=\hat{T}_{\infty}+\hat{T}(\omega)
$$

where $\hat{T}_{\infty}=\hat{\mathcal{T}}(\omega=\infty)$. The function $T_{\mathbf{q q}^{\prime}}(\omega)$ is analytical in the upper half-plane, hence, the following Lehmann representation 26 holds for it

$$
\hat{T}(\omega)=-\frac{1}{\pi} \int_{-\infty}^{\infty} d \omega^{\prime} \hat{T}^{\prime \prime}\left(\omega^{\prime}\right) /\left(\omega-\omega^{\prime}+i 0\right),
$$

where $\hat{T}^{\prime \prime}(\omega)=\operatorname{Im}\{\hat{T}(\omega)\}$. Sustituting Eqs. (5) - (7) into Eq. (4) and performing integration over $\omega^{\prime}$ by closing the integration path in the lower half-plane, one gets after some algebra

$$
\begin{gathered}
A_{\mathbf{q}^{\prime} \mathbf{q}}(t)=\frac{1}{\varepsilon\left(\mathbf{q}^{\prime}\right)-\varepsilon(\mathbf{q})}\left\{\left(\hat{T}_{\infty}\right)_{\mathbf{q}^{\prime} \mathbf{q}}\left[\frac{\exp \left(-i \varepsilon\left(\mathbf{q}^{\prime}\right) t\right)}{\omega-\varepsilon\left(\mathbf{q}^{\prime}\right)+i \alpha}-\frac{\exp (-i \varepsilon(\mathbf{q}) t)}{\omega-\varepsilon(\mathbf{q})+i \alpha}\right]-\frac{1}{\pi} \int_{-\infty}^{\infty} d x T_{\mathbf{q}^{\prime} \mathbf{q}}^{\prime \prime}(x)\right. \\
\left.\times\left[\frac{1}{x-\varepsilon\left(\mathbf{q}^{\prime}\right)}\left(\frac{\exp (-i x t)}{\omega-x+i \alpha}-\frac{\exp \left(-i \varepsilon\left(\mathbf{q}^{\prime}\right) t\right)}{\omega-\varepsilon\left(\mathbf{q}^{\prime}\right)+i \alpha}\right)-\frac{1}{x-\varepsilon(\mathbf{q})}\left(\frac{\exp (-i x t)}{\omega-x+i \alpha}-\frac{\exp (-i \varepsilon(\mathbf{q}) t)}{\omega-\varepsilon(\mathbf{q})+i \alpha}\right)\right]\right\} .
\end{gathered}
$$


Equation (8) provides an exact expression for the exciton wave function in the momentum representation. The wave function in the coordinate representation can be found by the Fourier transformation in the variable $\mathbf{q}^{\prime}$. This transformation can be performed only numerically and depends on the exciton dispersion law and the interaction potential between the exciton and the impurity. However, for optical applications this transformation is not needed. The photon scattering amplitude is completely determined by the amplitude $A_{\mathbf{q}^{\prime} \mathbf{q}}(t)$ in the small $\mathbf{q}$ and $\mathbf{q}^{\prime}$ region. Under these conditions the energies $\varepsilon(\mathbf{q})$ and $\varepsilon\left(\mathbf{q}^{\prime}\right)$ are nearly equal. For $\mathbf{q}^{\prime}, \mathbf{q} \rightarrow 0$ both the numerator and denominator vanish and Eq. (8) takes the form

$$
\begin{aligned}
A(t) & =T_{\infty} \frac{d}{d \varepsilon}\left(\frac{\exp (-i \varepsilon t)}{\omega-\varepsilon+i \alpha}\right) \\
& -\frac{1}{\pi} \int_{-\infty}^{\infty} d \omega^{\prime} T^{\prime \prime}\left(\omega^{\prime}\right) \frac{d}{d \varepsilon}\left[\frac{1}{\omega^{\prime}-\varepsilon}\left(\frac{\exp \left(-i \omega^{\prime} t\right)}{\omega-\omega^{\prime}+i \alpha}-\frac{\exp (-i \varepsilon t)}{\omega-\varepsilon+i \alpha}\right)\right] .
\end{aligned}
$$

Here $\varepsilon=\varepsilon(0)$ is the energy of long-wave-length excitons, and $A(t), T_{\infty}$, and $T^{\prime \prime}\left(\omega^{\prime}\right)$ are the limits of $A_{\mathbf{q}^{\prime} \mathbf{q}}(t),\left(T_{\infty}\right)_{\mathbf{q}^{\prime} \mathbf{q}}$, and $T_{\mathbf{q}^{\prime} \mathbf{q}}(\omega)$, respectively, for $\mathbf{q}, \mathbf{q}^{\prime} \rightarrow 0$. Equation (9) is the final equation for the time-dependent amplitude $A(t)$. It is completely determined by $T_{\infty}$ and the imaginary part of the operator $\hat{T}(\omega)$.

\section{FREE INDUCTION SIGNAL}

Equation (9) determines the time dependence of the free induction signal. Two basic properties of this equation follow from general arguments.

First, the derivatives $d / d \varepsilon$ result in a contribution to $A(t)$ proportional to $t \exp (-i \varepsilon t)$. This property is obvious as applied to the first and third terms of Eq. (9). A similar contribution from the second term can be found by using the identity $d\left(\omega^{\prime}-\varepsilon\right)^{-1} / d \varepsilon=$ $-d\left(\omega^{\prime}-\varepsilon\right)^{-1} / d \omega^{\prime}$ and transforming the integral by parts. The term in the amplitude $A(t)$

increasing with $t$ will be termed below as the growing mode.27 It originates from the product of two $G^{0}$ functions with nearly coinciding poles, Eq. (9). The growing mode is reminiscent of the growing solutions of differential equations with degenerate characteristic numbers. 
This mode describes the global evolution of the wave packet prepared by the pulse. As $t$ increases, the packet expands in $\mathbf{r}$ space. For translationally invariant systems this expansion is accompanied by changes in the phases of different Fourier components, whereas their moduli remain unchanged. The impurity potential, attractive or repulsive, violates the momentum conservation. Consequently, the amplitude of the $\mathbf{q}=0$ mode grows. It indicates that the volume of the coherence area around the impurity increases with $t$. The giant oscillator strengths observed in stationary experiments were ascribed by Thomas and Hopfield to exciton antennas. 28 In these terms the growing mode is generated by an inflating exciton antenna. This picture explains why the growing mode is specific for systems possessing a continuous spectrum and, hence, extended states. Mathematically this mode originates from the integration of $G_{\mathbf{q}^{\prime} \mathbf{q}}(\omega)$ along the cut in the complex plane passing across the exciton band.

Second, the bound state is a pole of $\hat{T}(\omega)$. Therefore, $T^{\prime \prime}\left(\omega^{\prime}\right)$ includes a term proportional to $\delta\left(\omega^{\prime}-\varepsilon+\varepsilon_{b}\right)$, where $\varepsilon_{b}$ is the binding energy of an exciton to the impurity center. This term contributes into Eq. (9) an oscillating exponent $\exp \left[-i\left(\varepsilon-\varepsilon_{b}\right) t\right]$. The exciton bandwidth is supposed to be large as compared with $\varepsilon_{b}$. Under these conditions the integration along the cut in the complex plane contributes the factor $\exp (-i \varepsilon t)$. Two oscillating terms in Eq. (9), $\exp \left[-i\left(\varepsilon-\varepsilon_{b}\right) t\right]$ and $\exp (-i \varepsilon t)$, result in beats at the frequency of $\varepsilon_{b}$ with a time-independent amplitude. It is remarkable that the oscillations remain undamped despite the fact that $\varepsilon$ belongs to a continuous spectrum. The scillating contribution to $A(\omega)$ will be termed the beating mode.

The scattering operator $\hat{\mathcal{T}}(\omega)$ can be easily found for a Frenkel exciton when the impurity potential is described by a degenerate perturbation $U_{\mathbf{m n}}=U \delta_{\mathbf{m} 0} \delta_{0 \mathbf{n}}, U<0.29$ Here $\mathbf{m}$ and $\mathbf{n}$ denote sites, and the impurity resides at the site $\mathbf{m}=\mathbf{n}=0$. Under these conditions $\mathcal{T}_{\mathbf{q}^{\prime} \mathbf{q}}(\omega)$ does not depend on the momenta $\mathbf{q}$ and $\mathbf{q}^{\prime}$ and equals

$$
\mathcal{T}(\omega)=\frac{U}{1-U G_{0}(\omega)}, \quad G_{0}(\omega)=\int \frac{\rho\left(\omega^{\prime}\right)}{\omega-\omega^{\prime}+i 0} d \omega^{\prime}
$$

where $G_{0}(\omega)$ denotes the free Green function $G_{\mathbf{q}}^{0}(\omega)$ integrated over the momentum $\mathbf{q}$, and 
$\rho(\omega)$ is the density of states inside the exciton band. It is easily seen that $T_{\infty}=U$. Binding energy $\varepsilon_{b}$ is related to the potential $U$ by the equation $G_{0}\left(\varepsilon-\varepsilon_{b}\right)=U^{-1}$. For a twodimensional (2D) system the density $\rho(\omega)$ can be modeled as $\rho(\omega)=1 / E_{B}$ for $0 \leq \omega \leq E_{B}$, where $E_{B}$ is the bandwidth, and the integral in Eq. (9) can be performed. Finally

$$
\mathcal{T}(\omega)=E_{B} /\left\{\ln \left[\frac{\varepsilon_{b}\left(E_{B}-\omega\right)}{\omega\left(E_{B}+\varepsilon_{b}\right)}\right]+i \pi\right\} .
$$

The amplitude $A(t)$ is shown in Fig. 1 for three values of $\omega$. Both the linear-in- $t$ growth and the oscillations with a time-independent amplitude are distinctly seen in the asymptotic region $t \gtrsim 2 \pi \varepsilon_{b}^{-1}$. Actually, they are seen even for small values of $t>0$, but the shape of the first oscillation is somewhat distorted. The data for a 3D system with a model density $\rho(\omega)=8 \sqrt{\omega\left(E_{B}-\omega\right)} / \pi E_{B}^{2}$ are also shown in Fig. 1. One can see that the dependence of $A(t)$ on dimensionality is rather weak. The linear-in- $t$ growth of $A(t)$ in the large $t$ region originates from the exciton dynamics. It disappears in the $E_{B} \rightarrow 0$ limit when the exciton effective mass tends to infinity.

Therefore, after a short transient the growing and beating modes dominate the amplitude $A(t)$. The optically detected free induction signal $I(t)$ is related to the zero-momentum component of the wave function, $I(t) \propto\left|\Psi_{0}(0, t)\right|^{2}$. In the asymptotic region

$$
A(t) \propto\left\{t+\frac{b}{2} \exp \left[i\left(\varepsilon_{b} t+\phi\right)\right]\right\} \mathrm{e}^{-i \varepsilon t}
$$

where $b$ and $\phi$ are real parameters, and $I(t)$ obeys the following law:

$$
I(t) \propto\left\{t^{2}+b t \cos \left(\varepsilon_{b} t+\phi\right)\right\}
$$

Interference of the two modes results in an unusual shape of the signal $I(t)$. It consists of the monotonic and oscillatory contributions, and both of them growing (rather than decaying) with $t .30$

Figure 11 displays the typical shape of the response $A(t)$. It is instructive to consider also a special case when a spectrally narrow pulse is in resonance with the bound-exciton state. The data are shown in Fig. 2. For $t \ll \alpha^{-1}$ the amplitude $|A(t)|$ is nearly constant 
and describes a bound exciton. As $t$ increases, the admixture of the continuum grows and the shape of the amplitude described by Eq. (12), linear growth and strong undamped oscillations, sets in for $t \gtrsim 3 \alpha^{-1}$. If the pulse is out of resonance with the bound exciton, the shape of the response similar to that of Fig. 1 is recovered.

The above theory does not take into account the radiative decay of the exciton wave packet. It is this approximation that results in the unlimited increase in the amplitude $A(t)$, Eq. (12). If one neglects the oscillating part of Eq. (13), the radiative lifetime $\tau_{R}(t)$ decreases with $t$ as $\tau_{R}(t) \propto t^{-2}$. This rapid increase in the emission probability implies a nonLorentzian shape of the emission signal and establishes the applicability limit for Eq. (13). A rigorous approach should include a generalization of the Weisskopf-Wigner theory as applied to the exciton antenna. We restrict ourselves to a phenomenological approach, which allows us to estimate the duration of the emitted pulse.

One can infer from Fig. 1 that if $\alpha \sim \varepsilon_{b}$ and the carrier frequency $\omega$ of the exciting pulse is not far from the exciton band bottom $\varepsilon$, the radiative lifetime $\tau_{R}(t)$ can be evaluated as

$$
\tau_{R}^{-1}(t) \approx\left(\tau_{R}^{0}\right)^{-1}\left(1+\beta \varepsilon_{b} t\right)^{2}
$$

where the coefficient $\beta \sim 1$. The wave packet size at $t=0$ is approximately equal to the bound-exciton radius, hence, $\tau_{R}^{0}$ can be estimated as the bound-exciton radiative lifetime. Since a single light quantum should be radiated in the emitted pulse, the duration $\tau_{\mathrm{em}}$ of this pulse can be estimated from the equation

$$
\int_{0}^{1} d n=\int_{0}^{\tau_{\mathrm{em}}} d t / \tau_{R}(t)=1 .
$$

Equations (14) and (15) result in the following formula for $\tau_{\mathrm{em}}$

$$
\tau_{\mathrm{em}} \approx\left(\tau_{R}^{0} / \varepsilon_{b}^{2}\right)^{1 / 3}
$$

which is correct with the accuracy to a numerical factor, which hopefully is approximately equal to unity. The number of oscillations within the emitted pulse is

$$
N \approx \tau_{\mathrm{em}} \varepsilon_{b} / 2 \pi \approx\left(\tau_{R}^{0} \varepsilon_{b}\right)^{1 / 3} / 2 \pi
$$


It is remarkable that, as distinct from the radiative lifetimes of atomic systems, $\tau_{\mathrm{em}}$ is proportional to $c$ rather than to $c^{3}$; here $c$ is the speed of light. This observation implies that $\tau_{\mathrm{em}}$ is shorter than the atomic radiative lifetimes by the factor $(1 / 137)^{2}$, i.e., $\tau_{\mathrm{em}} \sim 10^{-12}$ S.

It follows from Eq. (16) that the pulse is much shorter than $\tau_{R}^{0}$. For example, if $\tau_{R}^{0} \approx 1$ ns and $\varepsilon_{b} \approx 10 \mathrm{meV}$, the number of oscillations equals $N \approx 4$ and the pulse duration is $\tau_{\mathrm{em}} \approx 1.5 \mathrm{ps}$ in reasonable agreement with the above estimate.

Therefore, the inflating antenna shows an ultrashort radiative decay time $\tau_{\text {em }}$ having a picosecond scale. If $\tau_{\mathrm{em}} \ll \tau$, all scattered photons are emitted as a single burst, a short pulse having the shape of a train of oscillations. If $\tau_{\mathrm{em}} \gg \tau$, only a small number of photons are radiated in a short pulse of the duration about $\tau$, and the emission should show a rather long tail.

In all of the above a somewhat artificial shape of the exciting pulse, Eq. (1), was used. Nevertheless, the results are quite general. Indeed, the theory is linear in $\mathbf{E}_{\mathbf{q}}(\mathbf{r}, t)$, and a single-side exponential pulse can be considered as a Laplace component of a real pulse. Therefore, the free induction signal generated by an arbitrary pulse can be found by integration of Eq. (9) over $\alpha$ with a corresponding weighting factor. This integration does not change the general shape of the signal. The effect of smearing the sharply pointed pulse edge can be evaluated in a somewhat different way. If the termination points $t_{0}$ of exponential pulses are distributed according the Gaussian law $(\gamma / \sqrt{\pi}) \exp \left(-\gamma^{2} t_{0}^{2}\right)$, the first term in Eq. (12) remains unchanged, while the second term acquires the factor $\exp \left(-\varepsilon_{b}^{2} / 4 \gamma^{2}\right)$. If $\gamma \gtrsim \varepsilon_{b} / 2$, this factor results in the renormalization of the coefficient $b$ by a factor of about unity. It is of importance that the shape of the exciting pulse enters into the theory only as the initial condition, while the exotic behavior of the signal established above originates from the free evolution of the wave packet in the field of a defect. 


\section{FREE INDUCTION FROM BIEXCITONS}

In this section we generalize the above results for biexcitons. There exist two processes that result in optical production of biexcitons. 202,21,31 The first process is two-step absorption with an exciton level as a real intermediate state. In this process an exciton produced at the first step acts as an "impurity." All above results are applicable to this process without any serious changes. The second process is two-phonon absorption from the ground state. The theory of this process is more cumbersome than for impurity absorption. Nevertheless, the final results are nearly identical.

Biexciton eigenfunctions can be written in the operator form as

$$
|\mathbf{K} j\rangle=\frac{1}{\sqrt{2}} \int \frac{d \mathbf{k}}{(2 \pi)^{3}} \psi_{j}(\mathbf{k}) \psi_{\mathbf{K} / 2+\mathbf{k}}^{\dagger} \psi_{\mathbf{K} / 2-\mathbf{k}}^{\dagger}
$$

where $\psi_{\mathbf{K} / 2 \pm \mathbf{k}}^{\dagger}$ are exciton creation operators, and $\mathbf{K}$ is the center-of-mass momentum of a biexciton. Functions $\psi_{j}(\mathbf{k})$ are biexciton eigenfunctions in the momentum representation. The wave function of a biexciton wave packet at positive times can be found in the second order of the perturbation theory in the field $\mathbf{E}_{\mathbf{q}}(\mathbf{r}, t)$, Eq. (11). In the momentum representation, the wave function of the biexciton wave packet with a total momentum $\mathbf{K}=2 \mathbf{q}$ equals

$$
\Psi_{\mathbf{q}}(\mathbf{k}, t)=\frac{2 M^{2} \sqrt{V} / v}{\omega-\varepsilon(\mathbf{q})+i \alpha} A_{k 0}(t), \quad A_{k 0}(t)=\sum_{j} \frac{\psi_{j}(\mathbf{k}) \bar{\psi}_{j}(0)}{2 \omega-E_{j}+2 i \alpha} \mathrm{e}^{-i E_{j} t} .
$$

Here $E_{j}$ are energy levels of a two-exciton system, and $V$ is the normalization volume. If biexcitons are excited by two light beams with momenta $\mathbf{q}_{1}$ and $\mathbf{q}_{2}$, function $\bar{\psi}_{j}(0)$ in Eq. (19) should be substituted by $\bar{\psi}_{j}\left(\left(\mathbf{q}_{1}-\mathbf{q}_{2}\right) / 2\right)$.

Equation (19) differs from Eq. (2) only in the coefficient and in the change in variables, $\omega \rightarrow \Omega=2 \omega$ and $\alpha \rightarrow 2 \alpha$. Therefore, the transformations that led us from Eq. (2) to Eq. (9) can be repeated for biexcitons step by step. The equation for the scattering operator $\mathcal{T}_{\mathbf{k}^{\prime} \mathbf{k}}(\Omega)$ depends on the interaction between excitons. The zero-radius potential provides a satisfactory approximation for an exciton molecule. With this potential, the operator $\hat{\mathcal{T}}(\Omega)$ for a $3 \mathrm{D}$ continual system has the form 25 


$$
T_{\infty}=0, \quad T(\Omega)=\frac{a / 4 \pi^{2} m}{1+i a \sqrt{2 m(\Omega+i 0)}} .
$$

Here $m$ is the exciton mass, and $\sqrt{\Omega+i 0}$ has a positive imaginary part. Biexcitons exist when $T(\Omega)$ has a pole, i.e., for $a>0$; the scattering length $a$ equals $a=\left(2 m \varepsilon_{b}\right)^{-1 / 2}$, where $\varepsilon_{b}$ is the biexciton binding energy. Similarly to $\mathcal{T}(\omega)$ of Eq. (11), the operator $\mathcal{T}(\Omega)$ of Eq. (20) does not depend on momenta. Finally, $A(t)$ shows actually the same behavior as for bound excitons.

One can infer from Eq. (18) that the quantum state $\Psi_{\mathbf{q}}$ decays into two photons with momenta $\mathbf{K} / 2 \pm \mathbf{k}$. Intensity of the free induction signal is proportional to $\left|\Psi_{\mathbf{q}}(\mathbf{k}, t)\right|^{2} \approx$ $\left|\Psi_{0}(0, t)\right|^{2}$. In the asymptotic region $A(t) \propto\left\{t+(b / 2) \exp \left[i\left(\varepsilon_{b} t+\phi\right)\right]\right\} \exp (-2 i \varepsilon t)$, and Eq. (13) describes the optically detected signal.

Therefore, the existence of the growing and beating modes in the free induction amplitude is a common property of biexcitons and bound excitons 32 Estimates for the pulse duration, $\tau_{\text {em }}$, derived in Sec. [1] for bound excitons, are also applicable to biexcitons.

\section{DISCUSSION}

In this paper a theory of transients for biexcitons (bound excitons) is developed. It is a distinctive feature of the theory that the continuous spectrum and the exciton-exciton (exciton-impurity) interaction were consistently taken into account. Equation (9) for the amplitude $A(t)$ and the asymptotic expansion, Eq. (12), were found by exact solution of a well-established model. These equations reveal the behavior of the free induction signal described in Sec. [II]. The theory is reliable while time $t$ is small as compared with the duration of the emitted pulse, $t<\tau_{\mathrm{em}}$. Under these conditions the theory describes the basic dynamics of exciton wave packets. The larger the number of oscillations inside the emitted pulse, $N$, the wider the applicability region of the theory.

It is the main restriction of the theory that the radiative decay of wave packets was taken into account in a phenomenological way. Numerical coefficients in Eqs. (16) and (17) for 
$\tau_{\text {em }}$ and $N$, respectively, were evaluated only approximately. Actually, the values of these coefficients depend on the number of parameters (carrier frequency of the exciting pulse, its spectral width, etc.). Therefore, the above restriction does not influence the specific predictions of the theory, but impedes establishing rigorous criteria of its applicability.

The problem of the radiative decay is also closely related to the polariton concept. It is known that in 3D polariton effects do not change the results critically if the longitudinaltransverse splitting, $\Delta_{l t}$, and the size of the coherence region are small in comparison with $\varepsilon_{b}$ and the light wavelength $\lambda$, respectively. We do not know of any experimental data on the gigantic oscillator strengths of bound excitons that reveal deviations from the elementary theory 19 neglecting polariton effects. Apparently, the polariton theory 33 of this effect has never been applied for treating experimental data. Polariton theory of the biexciton spectra was also devoloped, 4,55 and it was only recently that the detection of the polariton contribution to the giant two-photon biexciton absorption in $\mathrm{CuCl}$ has been claimed 35 The polariton contribution to the inflating antenna theory should become of critical importance for large values of $t$ when the size of the antenna, $d(t)$, approaches $\lambda$. Apparently, the criteria $d(t)<\lambda$ and $t<\tau_{\mathrm{em}}$ impose similar restrictions on $t$. Therefore, a consistent theory of the radiative decay of the inflating antenna should be based on the polariton concept. The role of polaritons depends on dimensionality, and in lower dimensionalities their effect increases 36 For such systems the application on the polariton concept might be of special importance.

In conclusion, the coexistence of the continuous spectrum and a bound state results in growing and beating modes in the free induction signal following the exciting pulse, for both biexcitons and bound excitons. The duration of the free induction signal is controlled by the radiative decay rate and dephasing. If the first mechanism dominates, the signal is emitted as a short burst and the radiative yield is close to unity. 


\section{ACKNOWLEDGMENTS}

I am grateful to M. D. Sturge and J. M. Worlock for suggestive discussions and critical reading the manuscript. The support of the Office of Naval Research under Contract No. N000149410853 is acknowledged. 


\section{REFERENCES}

${ }^{1}$ Y. Masumoto, S. Shionoya, and T. Takagahara, Phys. Rev. Lett. 51, 923 (1983); L. Schultheis, M. D. Sturge, and J. Hegarty, Appl. Phys. Lett. 47, 995 (1985); L. Schultheis, J. Kuhl, A. Honord, and C. W. Tu, Phys. Rev. Lett. 57, 1797 (1986); B. Fluegel et al., ibid. 59, 2588 (1987).

${ }^{2}$ G. E. Pikus and E. L. Ivchenko, in Excitons, edited by E. I. Rashba and M. D. Sturge (North-Holland, Amsterdam, 1982).

${ }^{3}$ V. Langer, H. Stolz, and W. von der Osten, Phys. Rev. Lett. 64, 854 (1990).

${ }^{4}$ E. O. Göbel et al., Phys. Rev. Lett. 64, 1801 (1990).

${ }^{5}$ B. F. Feuerbacher, J. Kuhl, R. Eccleston, and K. Ploog, Solid State Commun. 74, 1279 (1990).

${ }^{6}$ K. Leo, T. C. Damen, J. Shah, and K. Köhler, Phys. Rev. B 42, 11359 (1990).

${ }^{7}$ S. Bar-Ad and I. Bar-Joseph, Phys. Rev. Lett. 68, 349 (1992); D. J. Lovering, R. T. Phillips, G. J. Denton, and G. W. Smith, ibid. 68, 1880 (1992); B. Oberhauser et al., Phys. Stat. Sol. B 173, 53 (1992).

${ }^{8}$ H. Wang et al., Solid State Commun. 98, 807 (1996).

${ }^{9}$ S. Weiss et al., Phys. Rev. Lett. 69, 2685 (1992); D.-S. Kim et al., ibid. 69, 2725 (1992).

${ }^{10}$ H. Wang, J. Shah, T. C. Damen, and L. N. Pfeiffer, Solid State Commun. 91, 869 (1994).

${ }^{11}$ S. Schmitt-Rink and D. S. Chemla, Phys. Rev. Lett. 57, 2752 (1986).

12 S. Schmitt-Rink, D. S. Chemla, and H. Haug, Phys. Rev. B 37, 941 (1988).

${ }^{13}$ M. Lindberg, R. Binder, and S. W. Koch, Phys. Rev. A 45, 1865 (1992), and references therein.

14 T. Östreich, K. Schönhammer, and L. J. Sham, Phys. Rev. Lett. 74, 4698 (1995). 
${ }^{15}$ S. Glutsch, U. Siegner, and D. S. Chemla, Phys. Rev. B 52, 4941 (1995).

${ }^{16}$ G. Finkelstein, S. Bar-Ad, O. Carmel, I. Bar-Joseph, and Y. Levinson, Phys. Rev. B 47, 12964 (1993); T. Saiki, M. Kuwata-Gonokami, T. Matsusue, and H. Sakaki, ibid. B 49, 7817 (1994).

${ }^{17}$ K. Bott et al., Phys. Rev. B 48, 17418 (1993).

${ }^{18}$ R. Zimmermann, Il Nuovo Cimento 17D, 1801 (1995); D. S. Citrin, Phys. Rev. B 54, 14572 (1996); S. Haake, R. A. Taylor, R. Zimmermann, I. Bar-Joseph, and B. Deveaud, Phys. Rev. Lett. 78, 2228 (1997).

${ }^{19}$ E. I. Rashba, Opt. Spektrosk. 2, 568 (1957); E. I. Rashba and G. E. Gurgenishvili, Fiz. Tverd. Tela 4, 1029 (1962) [Sov. Phys. Solid State 4, 1029 (1962)].

${ }^{20}$ A. A. Gogolin and E. I. Rashba, Pis'ma Zh. Eksp. Teor. Fiz. 17, 690 (1973) [JETP Lett. 17, $478(1973)]$.

${ }^{21}$ E. Hanamura, Solid State Commun. 12, 951 (1973).

${ }^{22}$ Critical role of the interexciton interaction in the two-exciton continuum manifests itself in recent experiments by S. T. Cundiff, M. Koch, W. H. Knox, J. Shah, and W. Stolz, Phys. Rev. Lett. 77, 1107 (1996).

${ }^{23}$ J. L. Birman, in Excitons (Ref. 2), p. 27; E. S. Koteles, ibid., p. 83; E. L. Ivchenko, ibid., p. 141.

${ }^{24}$ S. T. Cundiff, H. Wang, and D. G. Steel, Phys. Rev. B 46, 7248 (1992).

${ }^{25}$ A. I. Baz', Ya. B. Zel'dovich, and A. M. Perelomov, Scattering, Reactions, and Decays in Nonrelativistic Quantum Mechanics (Wiley, New York, 1975).

${ }^{26}$ A. A. Abrikosov, L. P. Gor'kov, and I. E. Dzyaloshinskii, Methods of Quantum Field Theory in Statistical Physics (Prentice Hall, Englewood Cliffs, NJ, 1963). 
27 There exist also dimensionality dependent logarithmic corrections to the growing mode. They originate from the integration near $\omega^{\prime}=\varepsilon$ in Eq. (9). We will not discuss these corrections here.

${ }^{28}$ D. G. Thomas anf J. J. Hopfield, Phys. Rev. 175, 1021 (1968).

${ }^{29}$ V. L. Broude, E. I. Rashba, and E. F. Sheka, Spectroscopy of Molecular Excitons (Springer, Berlin, 1985).

${ }^{30}$ Lindberg et al. (Ref. 13) have found a linear-in- $t$ term in their theory of nonlinear responses based on semiconductor Bloch equations. This nonlinear polarization, which develops from the space-homogeneous waves excited by two pump pulses, has a different origin from the growing mode of Eq. (12). We attribute their result to a classical process of the transformation of two strong $\mathbf{k}_{1}$ and $\mathbf{k}_{2}$ waves into a $2 \mathbf{k}_{2}-\mathbf{k}_{1}$ signal driven by the electon nonlinearity. While the $2 \mathbf{k}_{2}-\mathbf{k}_{1}$ signal is still weak, its amplitude is proportional to $t$.

31 J. B. Grun, B. Hönerlage, and R. Levy, in Excitons (Ref. 2), p. 459.

32 There is an open question whether the phenomenon of inflating antenna exists in any form in dense exciton systems. If it does, it might be observed in Hartree-Fock data since that theory provides exciton amplitudes in the low-momentum region. The existence of an intimate connection between giant oscillator strengths and mean-field spectra has been established for molecular excitons (Ref. 29). At present one can only hypothesize whether the growing and oscillating four-wave-mixing signal found in Ref. 14 is related to inflating antenna.

${ }^{33}$ V. I. Sugakov, Opt. Spektrosk. 24, 477 (1968) [Opt. Spectrosc. 24, 253 (1968)]; J. J. Hopfield, Phys. Rev. 182, 945 (1969).

${ }^{34}$ A. A. Gogolin, Fiz. Tverd. Tela 15, 2746 (1973) [Sov. Phys. Solid State 15, 1824 (1974)].

${ }^{35}$ A. L. Ivanov, M. Hasuo, N. Nagasawa, and H. Haug, Phys. Rev. B 52, 11017 (1995), and references therein. 
${ }^{36}$ L. C. Andreani, F. Tassone, and F. Bassani, Solid State Commun. 77, 641 (1991); D. S. Citrin, Phys. Rev. B 50, 17655 (1994); A. L. Ivanov and H. Haug, Phys. Rev. Lett. 74, 438 (1995), and references therein. 


\section{FIGURES}

FIG. 1. Time dependence of the amplitude $|A(t)|$ for $\alpha=\varepsilon_{b}, E_{B}=10 \varepsilon_{b}$. Momentum $\mathbf{k}=0$ is at the bottom of the exciton band. 3D: solid lines, 2D: dotted lines. (1) $\omega-\varepsilon=0$, (2) $\omega-\varepsilon=-0.5 \varepsilon_{b}$, (3) $\omega-\varepsilon=-\varepsilon_{b}$. For $t \gtrsim 2 \pi / \varepsilon_{b}$ the amplitude shows a linear growth and undamped oscillations with a period $2 \pi / \varepsilon_{b}$.

FIG. 2. Time dependence of the amplitude $|A(t)|$ for a 3D system excited by a spectrally narrow pulse; $\alpha=0.03 \varepsilon_{b}, E_{B}=10 \varepsilon_{b}$. Momentum $\mathbf{k}=0$ is at the bottom of the exciton band. Solid line: $\omega-\varepsilon=-\varepsilon_{b}$; dotted line: $\omega-\varepsilon=-0.5 \varepsilon_{b}$. 


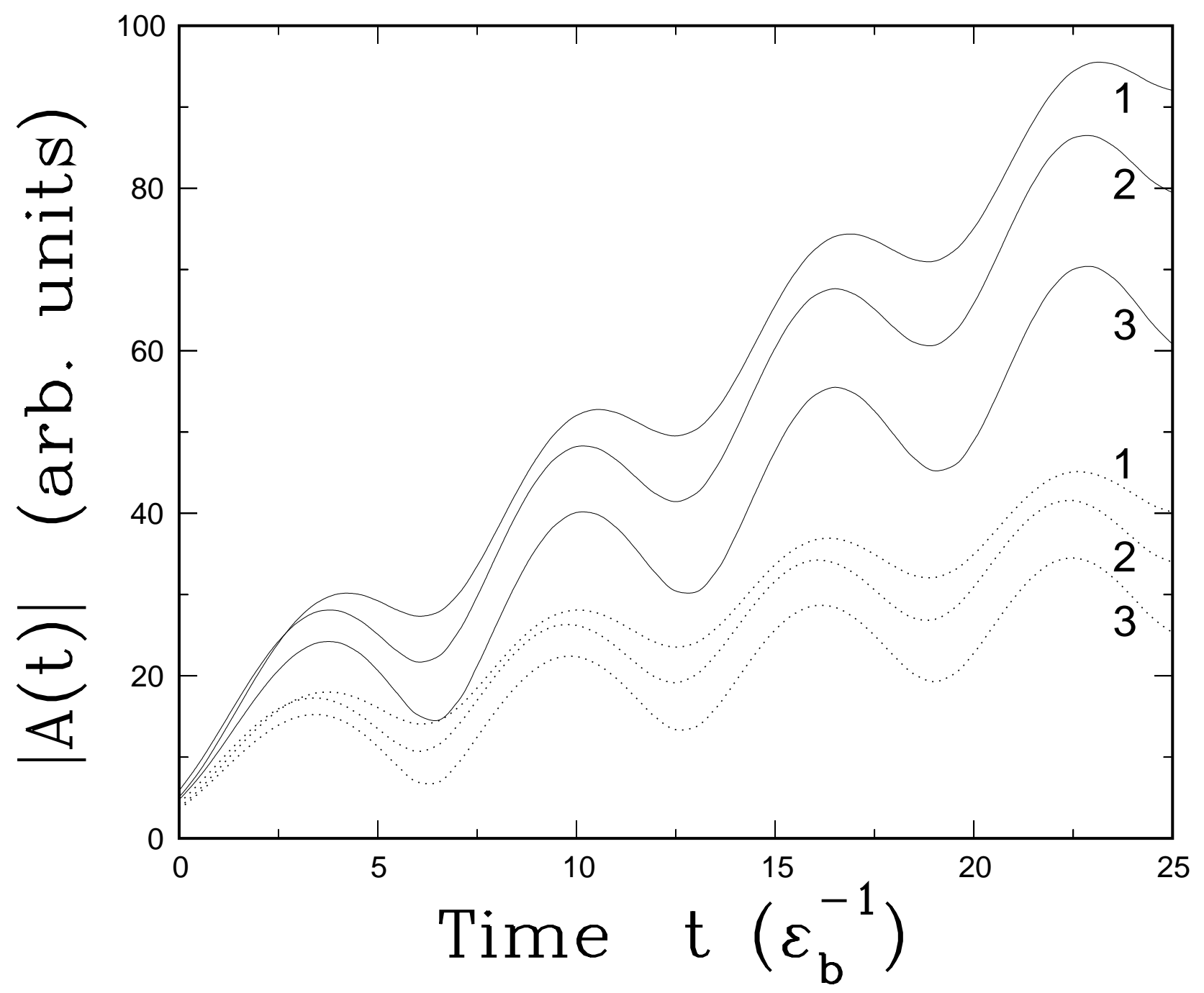




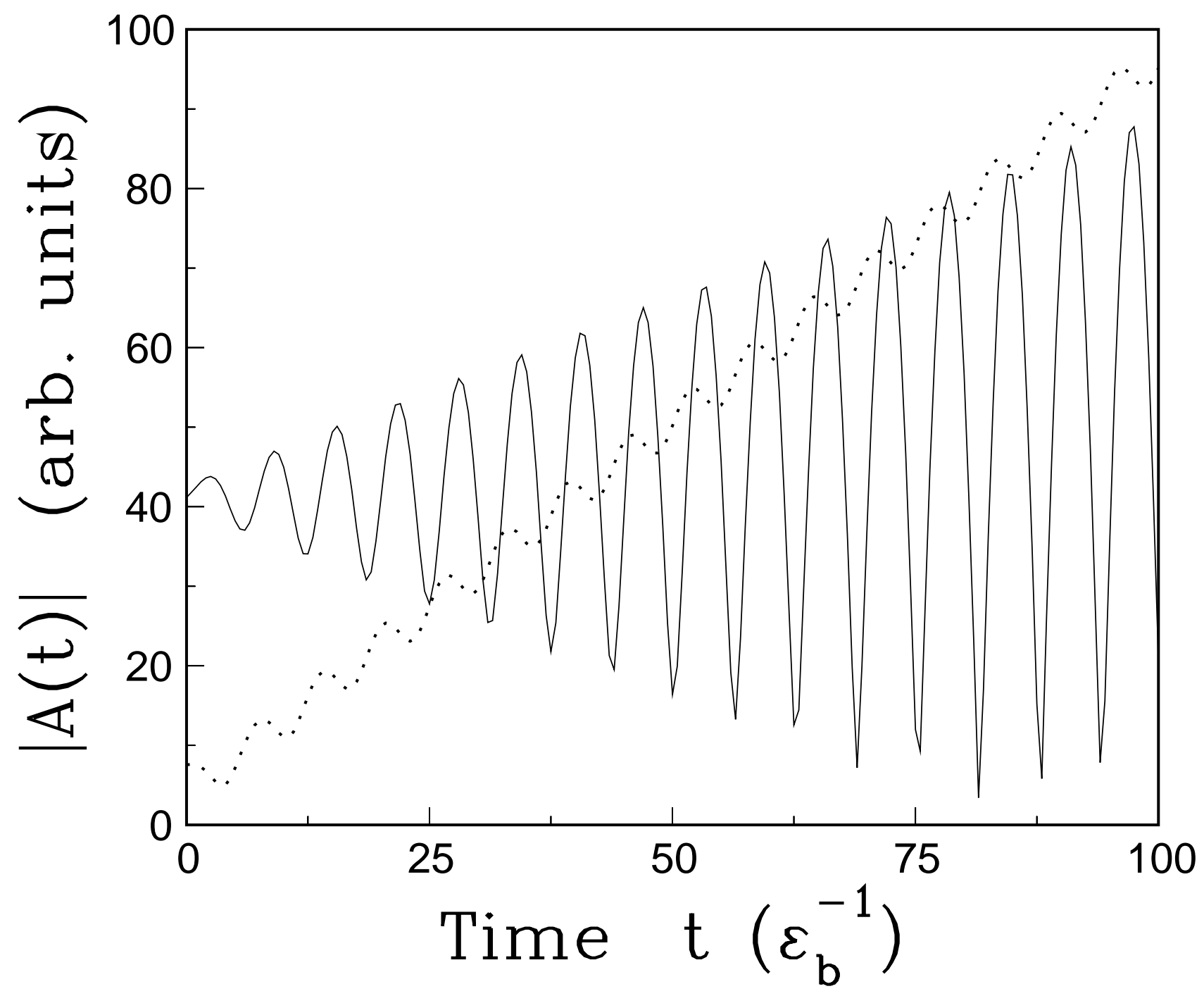

